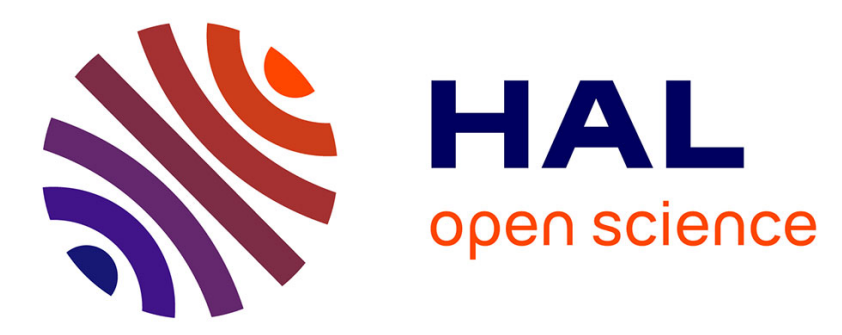

\title{
Modulation of phase separation at micron scale and nanoscale in hybrid polymer/lipid giant unilamellar vesicles (GHUVs)
}

\author{
Tuyen P. T. Dao, Fabio Fernandes, Emmanuel Ibarboure, Khalid Ferji, \\ Manuel Prieto, Olivier Sandre, Jean-François Le Meins
}

\section{To cite this version:}

Tuyen P. T. Dao, Fabio Fernandes, Emmanuel Ibarboure, Khalid Ferji, Manuel Prieto, et al.. Modulation of phase separation at micron scale and nanoscale in hybrid polymer/lipid giant unilamellar vesicles (GHUVs). Soft Matter, 2017, 13 (3), pp.627-637. 10.1039/C6SM01625A . hal-01417534

\author{
HAL Id: hal-01417534 \\ https://hal.science/hal-01417534
}

Submitted on 18 Nov 2018

HAL is a multi-disciplinary open access archive for the deposit and dissemination of scientific research documents, whether they are published or not. The documents may come from teaching and research institutions in France or abroad, or from public or private research centers.
L'archive ouverte pluridisciplinaire HAL, est destinée au dépôt et à la diffusion de documents scientifiques de niveau recherche, publiés ou non, émanant des établissements d'enseignement et de recherche français ou étrangers, des laboratoires publics ou privés. 
Received 17th July 2016, Accepted 5th December 2016

DOI: 10.1039/C6SM01625A

www.rsc.org/

\title{
Modulation of Phase Separation at micron scale and nanoscale in Hybrid Polymer/Lipid Giant Unilamellar Vesicles (GHUVs)
}

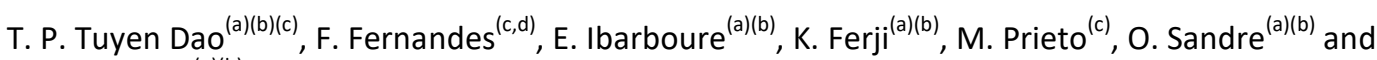 \\ J. F. Le Meins ${ }^{(a)(b)}$
}

Phase separation in hybrid polymer/lipid giant unillamelar vesicles (GHUVs) has been described over the last few years. However there is still a lack of understanding on the physical and molecular factors governing the phase separation in such systems. Among these parameters it has been suggested that in analogy to multicomponent lipid vesicles that hydrophobic mismatchs as well as lipid fludity play a role. In this work, we aim to map a global picture of phase separation and domain formation in membrane of GHUVs by using various copolymers based on poly(dimethylsiloxane) (PDMS) and poly(ethylene glycol) (PEO) with different architectures (grafted, triblock) and molar masses, combined with phospholipids at fluid (POPC) or gel state (DPPC) at room temperature. From confocal imaging and fluorescence lifetime imaging microscopy technique (FLIM), the phase separation into either micro- or nano- domains within GHVs were studied. In particular, our systematic studies demonstrate that in addition to lipid/polymer fraction or lipid physical state, important factors such as line tension at lipid polymer/lipid boundaries can be finely modulated by the molar mass and architecture of the copolymer and lead to the formation of stable lipid domains with different sizes and morphologies in such GHUVs.

\section{Introduction}

Mixing phospholipids and amphiphilic copolymers into a single hybrid membrane is a relatively recent approach developed in the past few years. Ideally, these structures could present biocompatibility and biofunctionality of liposomes, as well as robustness, low permeability and functionality variability conferred by the copolymer chains. This is expected to be of great interest in pharmaceutical applications for which only a few formulations based on liposomes are authorised on the drug market despite decades of research (e.g. DaunoXome ${ }^{\circledR}$, Doxil $^{\circledast} /$ Caelyx $^{\circledast}$ ) as well as in personal care. In particular, such moderate use of liposomes in clinics could be due to their lack of mechanical stability in the high shear rate of blood circulation through tiny vessels. Liposomal drug delivery system also often exhibits uncontrolled leakage phenomena (seen as a "burst release" effect on their pharmacokinetic profiles). As a consequence, the controlled release of encapsulated molecules at the pre-determined biological target (e.g. a tumour site) remains a difficult challenge. Besides the obvious interest of the association of lipids and amphiphilic copolymers into a single membrane of large unilamellar hybrid vesicles (LUHVs) for biomedical applications, the giant polymer/lipid hybrid unilamellar vesicles (GHUVs) can be also an excellent tool to get more insight into molecular and macroscopic parameter that govern the cell membrane

\footnotetext{
a. University of Bordeaux, LCPO UMR 5629, 16 avenue Pey Berland, F-33600 Pessac, France

${ }^{b .}$ CNRS, Laboratoire de Chimie des Polymères Organiques, UMR 5629, F-33600, Pessac, France

Centro de Química-Física Molecular and Institute of Nanoscience and Nanotechnology, Universidade de Lisboa Instituto Superior Técnico, 1049-001 Lisboa, Portugal

d. UCIBIO, REQUIMTE, Departamento de Química, Faculdade de Ciências e Tecnologia, Universidade Nova de Lisboa, 32829-516, Caparica, Lisbon, Portugal
}

Electronic Supplementary Information (ESI) available: NMR spectra and SEC curves of all synthesized triblock copolymers; Fluorescent images of hybrid polymer/lipid giant vesicles; Fluorescence single decay. See DOI: 10.1039/C6SM01625A domain formation, fusion and/or fission. In order to perfectly exploit the potential of such systems, the membrane structuration must be tuned either towards homogeneous mixing of the components or on the contrary to lateral phase separation, leading to the presence of nanoscale or micronsize domains. Then, the relationship between membrane structure and their physical and bio-functional properties must be better understood in order to eventually optimize them and validate their use in future biomedical applications, namely drug delivery, tumour targeting, bio-recognition, or bioadhesion.

Nevertheless, the literature on the subject is still relatively limited, ${ }^{1,2}$ although the scientific output is growing with interest from different scientific communities (biophysicists, biologists, physico-chemists). To date, the physical and molecular factors governing the phase separation in these hybrid polymer/lipid membranes are only partially understood. If phase separation occurring in multicomponent lipid bilayers is well documented since decades, ${ }^{3-12}$ research on hybrid polymer/lipid membrane is still in its infancy age. So far in the literature for both multicomponent lipid mixture and hybrid polymer/lipid mixture, the formation of membranes presenting a homogeneous distribution of components at least at the micrometric scale seemed to be favoured when the lipid was used above its main chain transition temperature whereas micron-scale lateral phase separation seems to be favoured below the lipid main transition temperature $\left(T_{\mathrm{m}}\right)$, where lipids are in a solid-ordered gel phase. ${ }^{3}$ It is important to note that in case of hybrid polymer/lipid vesicles, in addition to the expected chemical incompatibility between copolymer block chains and phospholipids, one has also to consider their respective dimensions. This point is particularly important as large differences in size can lead to a pronounced hydrophobic mismatch at the polymer/lipid boundaries. This height mismatch can be particularly pronounced in polymer/lipid mixtures. Hydrophobic mismatch leads to the exposure of hydrophobic groups to the aqueous environment which is 
This document is the Accepted Manuscript version of an article that appeared in final form in Soft Matter, 2017, 13(3), 627-637, after peer review and technical editing by the publisher. To access the final edited and published work see DOI: 10.1039/C6SM01625A

associated with a large energetic cost. This is likely to be one of the main contributors to line tension at domain boundaries. Thermodynamically, the line tension tends to favour domain coalescence (once a nucleation size is reached) to minimize the boundary length. ${ }^{13}$ As a consequence, the lipid domains would grow with time into one single large circular domain in the membrane. Theory also predicts another way to relax the energetic cost of membrane domains through the formation of "buds", by balancing the gain in free energy of lateral phase separation, the line tension, and the bending energy. ${ }^{14}$

In hybrid vesicles, neither the effect of polymer/lipid composition nor of hydrophobic mismatch on the domain formation was investigated in a systematic manner. Recent articles revealed that phase separation at micron scale can be obtained on GHUVs using copolymer forming vesicles with a membrane hydrophobic core thickness close to those of liposomes $(3 \sim 5 \mathrm{~nm})$, resulting in a relatively low hydrophobic mismatch, but also at nanoscale in LHUVs ${ }^{15,16}$. Many others studies used copolymers forming higher membrane thicknesses (at least $7 \mathrm{~nm}$ ), generally leading to homogeneous lateral structure at the micrometer scale ${ }^{17-21}$. From the results available so far, it seems that phase separation is less likely in the presence of a high hydrophobic mismatch between lipids and polymers.

When phase separation is obtained leading to micron size domains another key issue is their stability. Domain instability is driven by a balance between the bending energy of the domain and line tension at the boundaries. Edge energy can be decreased by reducing the perimeter of the circular domain through curvature and in this way increasing its bending energy. ${ }^{22-25}$ When the energetic cost associated with line tension is higher than the membrane bending energy required for fission, fission into separated vesicles can take place. This phenomenon has already been observed on hybrid polymerlipid membranes, above a critical lipid weight fraction, leading to separated liposomes and polymersomes. ${ }^{15,26}$

In this work, we aim to reach an overview of the parameters playing a role on phase separation as well as domain stability in hybrid polymer/lipid giant vesicles. The two main control parameters are hydrophobic mismatch and phospholipid fluidity. In addition, the influence of copolymer architecture has been also studied. For that purpose, we performed a systematic study over a whole composition range (from 0 to $100 \% \mathrm{w} / \mathrm{w}$ lipid) of the membrane structure of various GHUVs formed from a series of triblock copolymers based on poly(dimethylsiloxane) (PDMS) and poly(ethylene glycol) (PEO) with different molecular weights, allowing to modulate the polymer membrane thickness and therefore the hydrophobic length mismatch. The copolymers were combined with phospholipids at either fluid (POPC) or gel (DPPC) lipid phase. These lipids present comparable thickness of the hydrophobic bilayer in the fluid state $(3-3,5 \mathrm{~nm})^{27}$ The GHUVs were characterized through a combination of fluorescence microscopy and spectroscopy methodologies. The complex thermodynamics associated with hybrid polymer/lipid vesicles is confirmed by the observation of phase separation into micrometric domains, and/or nano-domains, eventually leading to budding or to complete fission for certain compositions.

\section{Materials and methods}

\section{Materials}

1-Palmitoyl-2-oleoyl-sn-glycero-3-phosphocholine (POPC); 1, 2dipalmitoyl-sn-glycero-3-phosphocholine (DPPC); 1,2-dioleoylsn-glycero-3-phosphoethanolamine- $\mathrm{N}$-(lissamine rhodamine $\mathrm{B}$ sulfonyl) (DOPE-Rhod) were obtained from Avanti Polar Lipids Inc., and used without further purification.

Amphiphilic graft copolymer $\mathrm{PDMS}_{26^{-}} g-\left(\mathrm{PEO}_{12}\right)_{2}$ was a gift from Dow Corning Inc. (\#5329) and thereafter denoted DOW. The characterizations of this copolymer were performed in the lab in a previous study ${ }^{28}$, all the results were in agreement with data given by the manufacturer and data available in literature 29,30 This copolymer form vesicles with a membrane thickness close to $5 \mathrm{~nm}$. Fluorescein-labelled graft copolymer $\left(\mathrm{PDMS}_{26^{-}} \mathrm{g}\right.$ $\left(\mathrm{PEO}_{12}\right)_{2}$-FITC) was prepared as previously described. ${ }^{15}$

Bovine Serum Albumin (BSA) was purchased from Aldrich. $\mathrm{B} \alpha$, $\omega$-bisaminopropyl-terminated poly(dimethylsiloxane) $\left(\mathrm{NH}_{2}-\right.$ PDMS- $\mathrm{NH}_{2}$ ) of different average molecular weight ( 1500,3000 and $5000 \mathrm{~g} / \mathrm{mol}$ ) were purchased from $A B C R$, Germany. Succinimidyl-((N-methyl)-poly ethyleneglycol) ester (509 and $686 \mathrm{~g} / \mathrm{mol}$ ) were purchased from Thermo Fisher Scientific Inc while $\alpha$-Methoxy- $\omega$-N-hydroxysuccinimide ester poly(ethylene glycol) $(750 \mathrm{~g} / \mathrm{mol}$ ) was from Rapp Polymer, Germany. Sucrose, glucose, and all organic solvents were purchased from Sigma Aldrich.

\section{Synthesis of Triblock Copolymers}

Various amphiphilic triblock copolymers $\mathrm{PEO}_{8}-b-\mathrm{PDMS}_{22}-b$ $\mathrm{PEO}_{8}, \mathrm{PEO}_{12}-b-\mathrm{PDMS}_{43}-b-\mathrm{PEO}_{12}$ and $\mathrm{PEO}_{17}-b-\mathrm{PDMS}_{67}-b-\mathrm{PEO}_{17}$ were synthesized via coupling $\alpha, \omega$-bisaminopropylterminated poly(dimethylsiloxane) $\left(\mathrm{NH}_{2}\right.$-PDMS- $\left.\mathrm{NH}_{2}\right)$ of different average molecular weight (1500, 3000 and 5000 $\mathrm{g} / \mathrm{mol})$ with Succinimidyl-((N-methyl)-ethyleneglycol) ester (509 and $686 \mathrm{~g} / \mathrm{mol}$ ) or $\alpha$-Methoxy- $\omega-\mathrm{N}$-hydroxysuccinimide ester poly(ethylene glycol) $(750 \mathrm{~g} / \mathrm{mol})$ (PEO-NHS). All molar masses have been checked by ${ }^{1} \mathrm{H} N M R$, leading respectively to 22, 43 and 67 units for the PDMS blocks and 8, 12 and 17 units for poly(ethylene oxide). The coupling reaction (Figure 1) was carried out under an inert gas in dichloromethane with the presence of DIPEA at room temperature during $24 \mathrm{~h}$. The copolymers were then purified by dialysis using membrane MWCO 50 kDa against Milli Q water during 2 days, dried by Iyophilisation, and characterized by NMR and GPC. (Supp Info Fig S1 and S2))

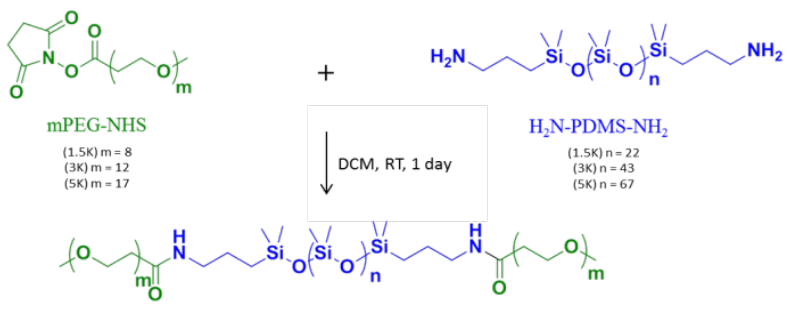

Figure 1. Synthesis scheme of PEG- $b$-PDMS- $b$-PEG triblock copolymer 
This document is the Accepted Manuscript version of an article that appeared in final form in Soft Matter, 2017, 13(3), 627-637, after peer review and technical editing by the publisher. To access the final edited and published work see DOI: 10.1039/C6SM01625A

\section{Hybrid giant unilamellar vesicles preparation}

All giant unilamellar vesicles in this study were prepared by the same process based on the electroformation method reported by Angelova, ${ }^{31}$ at room temperature for samples containing POPC, and at $50^{\circ} \mathrm{C}$ for samples containing DPPC. The probes were used at $0.2 \%$ molar for DOPE-Rhod and $1.5 \%$ molar for $\mathrm{PDMS}_{26}-\mathrm{g}-\left(\mathrm{PEO}_{12}\right)_{2}$-FITC. Briefly, mixtures of the appropriate lipids, polymers, and probes were prepared in chloroform/methanol solvent $(2 / 1 \mathrm{vol} / \mathrm{vol})$ at a total molar concentration of $1 \mathrm{mM}$. About $3 \mu \mathrm{L}$ of this solution was spread slowly on both sides of Pt electrodes. The electrodes were then submerged in sucrose solution at $100 \mathrm{mM}$ and immediately connected to an $\mathrm{AC}$ voltage. For either graft copolymer $\mathrm{PDMS}_{26^{-}} g-\left(\mathrm{PEO}_{12}\right)_{2}$ or triblock copolymers $\mathrm{PEO}_{8}-b$ $\mathrm{PDMS}_{22}-b-\mathrm{PEO}_{8}$ and $\mathrm{PEO}_{12}-b-\mathrm{PDMS}_{43}-b-\mathrm{PEO}_{12}$, a typical sinusoidal tension $(2 \mathrm{~V}, 1 \mathrm{~Hz})$ normally used for giant liposome preparation can be applied. For the $\mathrm{PEO}_{17}-b-\mathrm{PDMS}_{67}-b-\mathrm{PEO}_{17}$ triblock, a higher sinusoidal voltage $(10 \mathrm{~V}, 7 \mathrm{~Hz})$ had to be used. The vesicles were collected after 75 minutes by gently shaking the electrodes in the solution. Samples were kept at room temperature and in the dark until being used.

\section{Confocal imaging}

All measurements were performed on a Leica TCS SP5 (Leica Microsystems CMS GmbH, Mannheim, Germany) inverted confocal microscope (DMI6000). A $63 \times$ apochromatic water immersion objective with a NA of 1.2 (Zeiss, Jena Germany) was used for all experiments. $\left(\mathrm{PDMS}_{26}-\mathrm{g}-\left(\mathrm{PEO}_{12}\right)_{2}-\mathrm{FITC}\right)$ and DOPE-Rhod excitation were achieved with the $488 \mathrm{~nm}$ and 514 $\mathrm{nm}$ lines respectively from an argon laser while the emission was collected in the $500-530 \mathrm{~nm}$ range for $\left(\mathrm{PDMS}_{26^{-}} \mathrm{g}-\left(\mathrm{PEO}_{12}\right)_{2^{-}}\right.$ FITC) and in 600-700 $\mathrm{nm}$ range for DOPE-Rhod. Temperature control was achieved using a thermostated plate (Linkman, UK) mounted on the microscope stage.

\section{Fluorescence lifetime imaging microscopy}

Fluorescence lifetime imaging microscopy (FLIM) measurements were performed through time correlated single photon counting (TCSPC) using the same setup of confocal microscopy, coupled to a multiphoton Titanium: Sapphire laser (Spectra-Physics Mai Tai BB, Darmstadt, Germany, 710-990 $\mathrm{nm}, 100$ femtoseconds pulses, $80 \mathrm{MHz}$ ) as the excitation source. A photomultiplier tube was coupled to the X-port of the microscope and the emitted photons were processed by an SPC board that addresses simultaneously the $(x, y)$ location of the collected photons (Becker and Hickl, GmbH, PMC-100-4 SPC-830). The laser power was adjusted to give an average photon counting rate higher than $5.10^{4}$ photon/s and images were acquired during 60 seconds to achieve reasonable photon statistics.

The excitation wavelength was set to $820 \mathrm{~nm}$ and emission light was selected with a dichroic beam splitter with an excitation SP700 short-pass filter and an emission 530 bandpass filter inserted in front of the photomultiplier. Images were acquired using a Becker and Hickl SPC 830 module. Fluorescence lifetimes were obtained by analysing the fluorescence decays through a least square iterative reconvolution of decay functions with the instrument response function (IRF) using the software SPCImage (Becker and Hickl, Berlin, Germany).

\section{Micropipettes}

The evolution of the membrane structuration under controlled applied lateral tension was evaluated for some systems. Micropipettes were obtained by stretching Borosilicate capillaries (1mmOD, 0.58mmID) from WPI, with a pipette puller (Sutter Instrument P-97). The pulled pipets were then forged to the desired diameter using a micro-forge Narishige MF-900. Micropipettes were coated with BSA to prevent vesicle adhesion. The vesicle tension was controlled by a home-made hydraulic watertight setup, and micropipette was controlled by a micromanipulator (Eppendorf, Patchman NP2). The membrane tension was calculated classically from the Laplace equation:

$$
\sigma=\frac{\mathrm{P}_{\mathrm{s}} \times \mathrm{R}_{\mathrm{p}}}{2\left(1-\frac{\mathrm{R}_{\mathrm{p}}}{\mathrm{R}_{\mathrm{v}}}\right)}
$$

$\mathrm{R}_{\mathrm{p}}$ and $\mathrm{R}_{\mathrm{v}}$ are the micropipette and vesicle radius (outside the micropipette). $\mathbf{P}_{\mathbf{s}}$ is the suction pressure on the micropipette.

\section{Results}

\section{Amphiphilic triblock copolymers}

In order to study systematically the effect of polymer/lipid hydrophobic length mismatch on phase separation, different PEO- $b$-PDMS- $b$-PEO triblock copolymers varying in their block compositions were designed and synthesized. PDMS was chosen as the hydrophobic block because of its excellent biocompatibility as well as its low glass transition temperature, allowing dynamic exchanging of the chains and leading to the formation of membranes with a structure at thermal equilibrium. Moreover PDMS has been quite often used in the field to develop hybrid membranes ${ }^{15,17,32}$. It is worth mentioning that among the different hydrophobic block used for the formulation of GHUV (Polyisobutylene, ${ }^{33-35}$ PolyButadiene ${ }^{19,36,3720}$, PDMS presents the lower Hildebrand solubility parameters $\left(\delta=7.3 \mathrm{cal}^{1 / 2} / \mathrm{cm}^{3 / 2}\right)$ and would be therefore the less compatible with fatty acid tails in lipids $(\delta=$ $9.1 \mathrm{cal}^{1 / 2} / \mathrm{cm}^{3 / 2}$ ). The successful coupling of $\mathrm{NH}_{2}-\mathrm{PDMS}-\mathrm{NH}_{2}$ with PEO-NHS was verified by ${ }^{1} \mathrm{H}$ NMR through the disappearance of NHS group and the shift from $\delta=2.6 \mathrm{ppm}$ of proton near amine groups $\left(\mathrm{H}_{2} \mathrm{NCH}_{2} \mathrm{CH}_{2}-\right)$ to $\delta=3.1 \mathrm{ppm}$ of proton near amide groups ( $\mathrm{RCONHCH} \mathrm{CH}_{2}$ ) after reaction as illustrated in Figure S1. All molecular characterization by ${ }^{1} \mathrm{H}$ NMR and SEC of each individual obtained triblock copolymers were presented in section S1 and S2 respectively of supporting information. Their ability to self-assemble into vesicular structures and their corresponding membrane thicknesses were characterized by Cryo-TEM, as shown in Figure S6. These membrane thicknesses correspond to the thickness of the hydrophobic core, as PEO chains are not visible due to a weak electronic contrast. All characteristics of amphiphilic copolymers used in this study as well as the corresponding 
This document is the Accepted Manuscript version of an article that appeared in final form in Soft Matter, 2017, 13(3), 627-637, after peer review and technical editing by the publisher. To access the final edited and published work see DOI: 10.1039/C6SM01625A

membrane thicknesses of the polymersomes formed are described in Table 1.

\begin{tabular}{ccccc}
\hline Name & Composition & $\begin{array}{c}\mathrm{Mn}(\mathrm{g} / \mathrm{mol}) \\
\left({ }^{1} \mathrm{H} \mathrm{NMR}\right)\end{array}$ & $\oplus$ & $\begin{array}{c}\mathrm{d} \\
(\mathrm{nm})\end{array}$ \\
\hline DOW & $\mathrm{PDMS}_{26}-g-\left(\mathrm{PEO}_{12}\right)_{2}$ & 2750 & 1.32 & $5.6 \pm 0.6^{*}$ \\
$1.5 \mathrm{~K}$ & $\mathrm{PEO}_{8}-b-\mathrm{PDMS}_{22}-b-\mathrm{PEO}_{8}$ & 2668 & 1.18 & $5.4 \pm 0.4$ \\
$3 \mathrm{~K}$ & $\mathrm{PEO}_{12}-b-\mathrm{PDMS}_{43}-b-\mathrm{PEO}_{12}$ & 5014 & 1.28 & $8.8 \pm 0.5$ \\
$5 \mathrm{~K}$ & $\mathrm{PEO}_{17}-b-\mathrm{PDMS}_{67}-b-\mathrm{PEO}_{17}$ & 6700 & 1.23 & $11.2 \pm 1.2$ \\
\hline
\end{tabular}

Table 1. Molecular characteristics of amphiphilic copolymers using in this study. and corresponding membrane thickness $\mathrm{d}$. $*$ from ${ }^{15}$

\section{Micron scale fluid/fluid phase separation in GHVs}

In this section, we describe the tendency of micrometric fluid lipid domain formation for each mixture of copolymer/POPC through confocal microscopy measurements of GHUVs loaded with fluorescent derivatives of copolymer and phospholipids. Phase separation behaviour of GHUVs was monitored within a broad range of compositions (10-90\%) and temperatures (20$\left.55^{\circ} \mathrm{C}\right)$.

It is interesting to note that for all studied copolymers, electroformation of GHUVs was successful over the whole range of polymer/lipid fractions, although obviously some pure liposomes or polymersomes could be observed close to the $0 \%$ or $100 \%$ polymer compositions. All samples mentioned in this section were incubated at room temperature overnight after electroformation to reach an equilibration state before analysis through fluorescence confocal microscopy. The lipid, copolymer or hybrid nature of each domain is recognized from the presence of fluorescence from phospholipid (red channel) and/or copolymer (green channel) fluorescent derivatives, as illustrated on Figure 2.

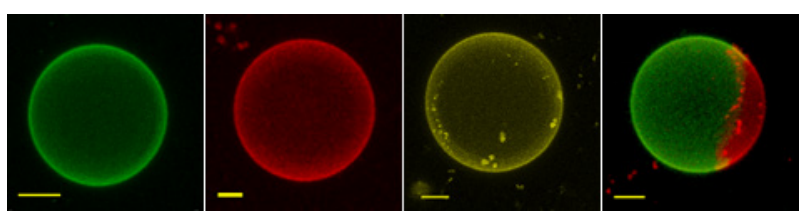

Figure 2. Overlay of maximum intensity $2 \mathrm{D}$ projection images taken for $\mathrm{PDMS}_{26} \mathrm{~g}$ $\left(\mathrm{PEO}_{12}\right)_{2}$-FITC (green channel) and DOPE-Rhod (red channel). From the left to the right: polymersome, liposome, mixed GHUV and demixed GHUV. Scale bar: $5 \mu \mathrm{m}$.

We first studied mixtures of lipid with $1.5 \mathrm{~K}$ copolymer which, in pure polymersomes, presents a membrane thickness comparable to the one of POPC, thus minimum hydrophobic length mismatch is expected. At room temperature, no macroscopic domains were observed in mixtures -at 10 weight $\%$ of POPC, showing a homogeneous distribution of both lipid and copolymer fluorescent analogues (Figure 3), as the polymer phase is likely able to integrate low concentrations of lipid homogeneously into the polymer-rich membrane. At a higher POPC content, formation of fluid lipid-rich domains is detected and above $25 \%$ weight POPC, most of the hybrid vesicles exhibit phase separation (Figure 3 ). It is also important to notice that above 50 weight \% of POPC, domain budding occurs. These budded domains were present both in lipid and copolymer-rich domains, and in many cases they remained stable at room temperature for at least two days after electroformation. Interestingly, a minor change in the boundary of the phase coexistence region is observed at higher temperatures and in some cases, lipid domains in GHUVs with low POPC concentrations (16\%, 25?\% wt/wt) at $20^{\circ} \mathrm{C}$ were no longer visible when temperature was increased. This is not expected as POPC is in the fluid phase for the whole range of temperatures studied $\left(20-55^{\circ} \mathrm{C}\right)$.

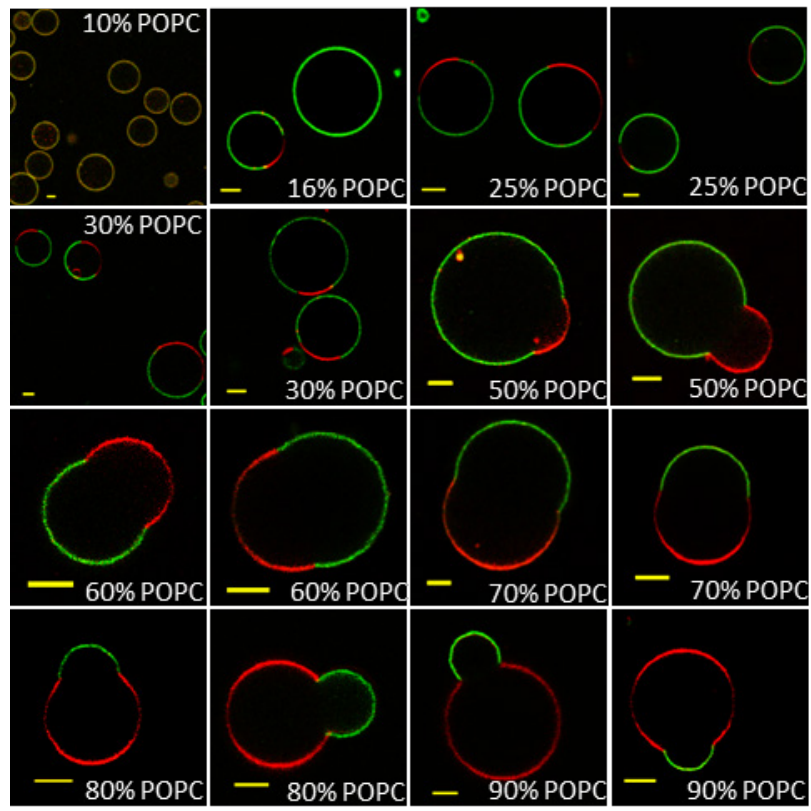

Figure 3. The equatorial slices of 1.5K/POPC GHUVs labeled with PDMS26-g-(PEO12)2FITC (green channel) and DOPE-Rhod (red channel) measured by confocal fluorescence microscopy at room temperature. Scale bar: $5 \mu \mathrm{m}$.

Additionally, temperature is shown to be crucial for the stability of budded domains, as at high POPC content $(80 \%$ $\mathrm{wt} / \mathrm{wt}$ ) and above $35^{\circ} \mathrm{C}$, budding domains were no longer visible and two GUV populations were now present, with either polymer-rich phases or lipid-rich homogeneous phases, suggesting that fission of coexisting domains is significantly more frequent at higher temperatures. Examples of fission events are illustrated in the Figure S8.

The same methodology was then also performed with the second triblock copolymer, $3 \mathrm{~K}$, forming vesicles with a membrane thickness higher than those of liposomes. In this case, homogeneous hybrid vesicles at the micrometre scale (yellow on the overlay image due to the presence of both DOPE-Rhod and PDMS $_{26}$-g-(PEO 12$)_{2}$-FITC fluorescence) were observed for lipid fractions up to $40 \%$ (wt/wt) of POPC (Figure 4). In addition, a fraction of pure liposomes was detected and vary with composition but corresponds approximately to $30 \%$ of total vesicle population for a $20 \%$ POPC mixture, slightly more pronounced compared to $1.5 \mathrm{~K}$ for which only $5 \%$ of pure liposomes were detected for this composition. Budding of domains became also more frequent occurring for the whole range of compositions where phase coexistence was observed. Those budded domains of 3K/POPC GHUVs also exhibited 
This document is the Accepted Manuscript version of an article that appeared in final form in Soft Matter, 2017, 13(3), 627-637, after peer review and technical editing by the publisher. To access the final edited and published work see DOI: 10.1039/C6SM01625A

fission into separated vesicles at high temperature as shown in Figure S10.

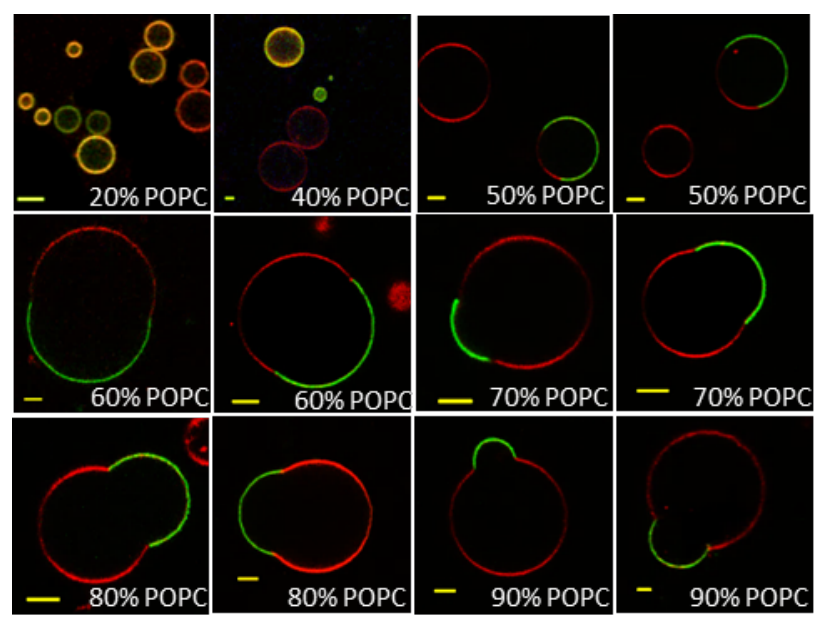

Figure 4. The equatorial slices of hybrid 3K/POPC GUVs labelled with $\mathrm{PDMS}_{26}-\mathrm{g}$ $\left(\mathrm{PEO}_{12}\right)_{2}$-FITC (green channel) and DOPE-Rhod (red channel) measured by confocal fluorescence microscopy at room temperature. Scale bar:Scale bar:Scale bar:Scale bar: $5 \mu \mathrm{m}$

Measurements with mixtures of $5 \mathrm{~K}$ copolymer with POPC led to different results. At lipid fractions as high as $50 \%$ and $75 \%$ (wt/wt), no phase coexistence was observed. With this significantly larger triblock copolymer chains, three populations of vesicles were systematically present: pure liposome, pure polymersome and a number of homogeneous structures. A representative image is shown in Figure S11. These results suggest that given the dramatic hydrophobic length mismatch between $5 \mathrm{~K}$ block chains and POPC, the existence of stable micrometric lipid enriched domains is not possible.

To support the hypothesis that conformational adaptation of the polymer chains to the smaller length of lipid molecules is the main control parameter, 3K/POPC hybrid vesicles were imaged immediately after electroformation, in a lipid fraction range where homogenous hybrid vesicles were predominantly observed. As can be clearly seen in the examples shown on Figure 5, GHUVs of 3K/POPC shortly after electroformation displayed curved domains which evolved towards fission over time and led to pure liposomes and originating vesicles in which the remaining lipid molecules were dispersed homogenously at the micron scale. This phenomenon also helps us explaining why a large number of pure liposomes were seen in 3K/POPC GHUVs as indicated above.

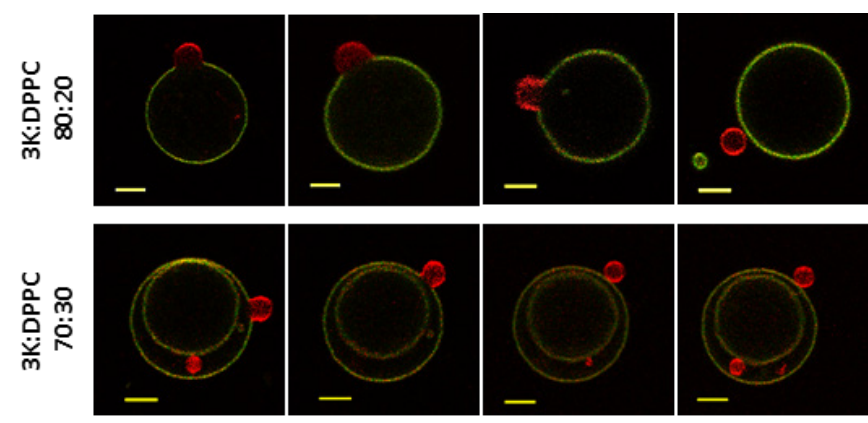

Figure 5. Typical situation observed just after electroformation for different GHUVs of 3K/POPC at low POPC concentrations: budding and fission of small lipid domains occurred quickly over time (order: left to right). Sscale bar: $5 \mu \mathrm{m}$.

\section{Nanodomain detection in GHVs}

For the detection of nanoscale phase separation, Förster resonance energy transfer (FRET) measurements were carried out through fluorescence lifetime imaging microscopy (FLIM) on copolymer/POPC hybrid vesicles.

The FRET efficiency between the polymer conjugate PDMS $_{26^{-}}$ $g$-(PEO $\left.{ }_{12}\right)_{2}$-FITC: FRET donor) and the labelled phospholipid (DOPE-Rhod: FRET acceptor) is extremely sensitive to phase separation, since the fluorescent polymer can only be incorporated into the polymer-rich phase while the lipid probe incorporates preferentially in the lipid-rich phases. ${ }^{16}$ Phase separation in GHUVs is expected to be the driving force of a decrease in FRET efficiency as the average distance between donors and acceptors increase. Experiments were made at polymer/lipid compositions for which no phase separation had been detected through confocal microscopy.

The $\mathrm{PDMS}_{26}-\mathrm{g}$ - $\left(\mathrm{PEO}_{12}\right)_{2}$-FITC fluorescence lifetime images of different GHUVs at different lipid contents are shown in figure 6. The corresponding histograms are also included for comparison. For pure $1.5 \mathrm{~K}$ polymersome, the lifetime histogram shows a homogeneous distribution centred at about 3 ns. When the acceptor probe is added, a large decrease of FITC fluorescence lifetime is observed as a consequence of a random distribution of the probes within the membrane. Without acceptor, when POPC was incorporated at increasing fractions $(5,10$ and $15 \% \mathrm{w} / \mathrm{w})$, the $\mathrm{PDMS}_{26^{-}} \mathrm{g}-\left(\mathrm{PEO}_{12}\right)_{2}-\mathrm{FITC}$ fluorescence lifetime distribution in those membranes became broader and slightly shifted to lower values. Fluorescence single decays were also measured for each GHUV (on at least 10-15 individual vesicles) and similar shifts were obtained (see supporting information Table S1. This dependence of $\mathrm{PDMS}_{26}{ }^{-}$ $g$-( $\left.\mathrm{PEO}_{12}\right)_{2}$-FITC fluorescence lifetime with lipid content is likely the result of changes in polymer phase properties due to the insertion of lipid molecules.

GHUVs loaded with $0.5 \%$ Rhod-DOPE as acceptor were then prepared for each of the above described $1.5 \mathrm{~K} / \mathrm{POPC}$ compositions. The fluorescence lifetime of $\mathrm{PDMS}_{26}-\mathrm{g}-\left(\mathrm{PEO}_{12}\right)_{2}-$ FITC was then recorded to assess FRET efficiencies. 
This document is the Accepted Manuscript version of an article that appeared in final form in Soft Matter, 2017, 13(3), 627-637, after peer review and technical editing by the publisher. To access the final edited and published work see DOI: 10.1039/C6SM01625A
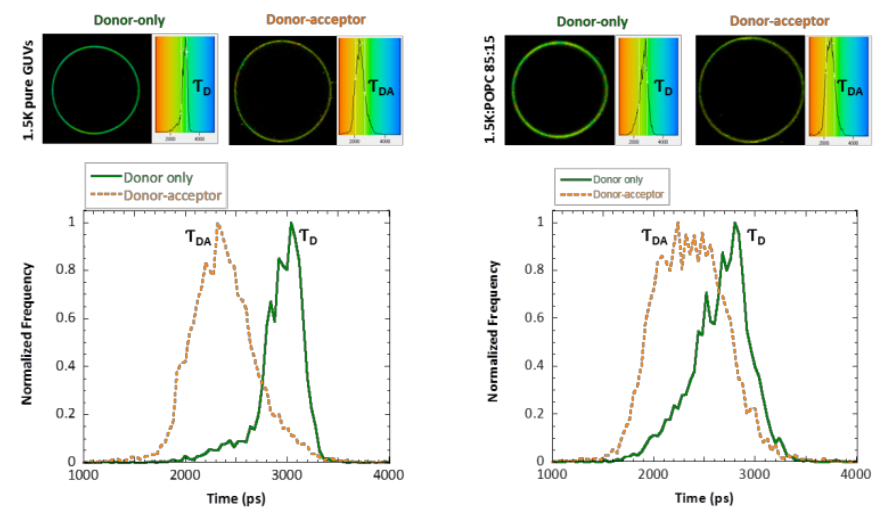

Figure 6. Equatorial z-section FLIM images and their representative $\mathrm{PDMS}_{26}$-g$\left(\mathrm{PEO}_{12}\right)_{2}$-FITC fluorescence lifetime distribution histograms. Left: pure $1.5 \mathrm{~K}$ GUVs labelled with $\mathrm{PDMS}_{26}-\mathrm{g}-\left(\mathrm{PEO}_{12}\right)_{2}$-FITC (FRET donor, $1.5 \%$ molar)) in both the presence and absence of Rhod-DOPE (FRET acceptor, 0.5\% molar). Right: GHUVs prepared from 1.5K/POPC 85/15 (wt/wt) mixtures labelled with $\mathrm{PDMS}_{26}-\mathrm{g}-\left(\mathrm{PEO}_{12}\right)_{2}$ FITC (FRET donor, 1.5\% molar)) in both the presence and absence of Rhod-DOPE (FRET acceptor, $0.5 \%$ molar).

The decrease of the $\mathrm{PDMS}_{26}-\mathrm{g}-\left(\mathrm{PEO}_{12}\right)_{2}$-FITC fluorescence lifetime was not as pronounced as the one observed in pure polymersomes where a homogeneous distribution was obviously expected. Interestingly, a clear decrease of FRET efficiencies with increasing POPC content was recorded, (Figure 6), indicating that the average distance between donor and acceptor probes increased as ascribed to nanoscale phase separation.

According to Monte Carlo simulations for distributions of donors and acceptors in a membrane environment under phase separation, a decrease in FRET efficiencies would only be noticeable if lipid domain dimensions were larger than the Förster radius of the donor-acceptor FRET pair (in this case $R_{0}$ $=5 \mathrm{~nm})^{38,39}$. In this way, nanoscale phase separation is evidenced at $1.5 \mathrm{~K} / \mathrm{POPC}$ mixtures for which no macroscopic (micron scale) phase separation was visible under confocal microscopy. Nanoscale phase separation occurs at a lipid content as low as $10 \% \mathrm{w} / \mathrm{w}$. At lower POPC concentrations, no FRET changes were observed, suggesting that either no phase separation took place or that lipid domains were smaller than $5 \mathrm{~nm}$ in diameter.

We followed exactly the same methodology for the 3K/POPC mixture. Once again, a strong decrease of fluorescence lifetime distribution was observed for pure polymersomes when the acceptor probe was added to the membrane, reflecting the random distribution of probes within the membrane (Figure 7). Without acceptor probe, we also observed a slight decrease in FITC fluorescence lifetime in 3K/POPC GUVs with increasing POPC content. When increasing POPC content, the decrease of lifetime was less pronounced in the presence of acceptor probe, compared to what was observed for pure polymersomes suggesting nanoscale phase separation in 3K/POPC GUVs. This hypothesis was also confirmed through FRET measurements in hybrid vesicles of lipid content lower than $40 \% \mathrm{wt} / \mathrm{wt}$ POPC as shown in Figure 8 . As seen for $1.5 \mathrm{~K} / \mathrm{POPC}$ mixtures, FRET efficiencies for the $\mathrm{PDMS}_{26}$-g-
$\left(\mathrm{PEO}_{12}\right)_{2}$-FITC / Rho-DOPE FRET pair, decreased with increasing POPC content.
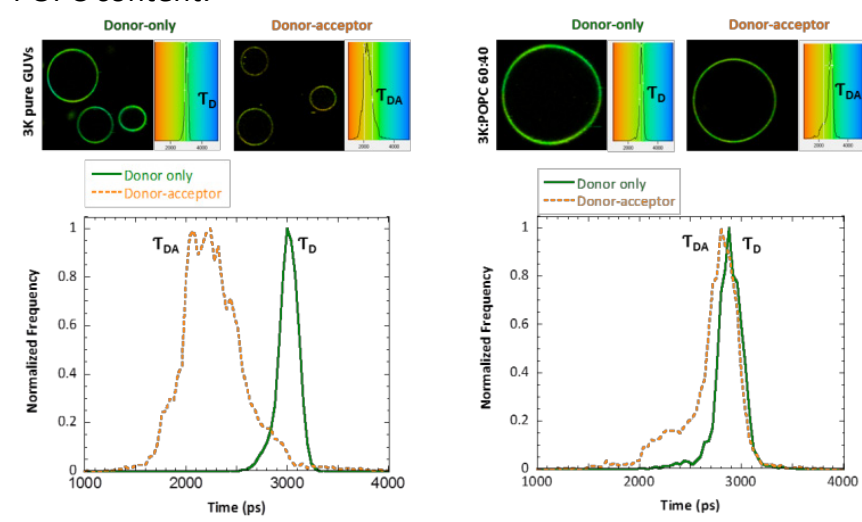

Figure 7. Equatorial z-section FLIM images and their representative $\mathrm{PDMS}_{26}$-g$\left(\mathrm{PEO}_{12}\right)_{2}$-FITC fluorescence lifetime distribution histograms. Left: pure 3K GUVs labelled with $\mathrm{PDMS}_{26}-\mathrm{g}-\left(\mathrm{PEO}_{12}\right)_{2}$-FITC (FRET donor, $1.5 \%$ molar)) in both the presence and absence of Rhod-DOPE (FRET acceptor, 0.5\% molar). Right: GHUVs prepared from 3K/POPC 60/40 (wt/wt) mixtures labelled with PDMS (6- $^{-}$- $-\left(\text {PEO }_{12}\right)_{2}$-FITC (FRET donor, 1.5\% molar)) in both the presence and absence of Rhod-DOPE (FRET acceptor, $0.5 \%$ molar).

At $40 \%$ POPC, the FRET was close to 0 with a large error bar. This result was unexpected, as part of the lipid goes into the polymer phase as suggested by our previous studies. However, as the lipid composition was not perfectly controlled in each of the vesicles, this may add to the uncertainty of the value (errors bars are significant).

Nevertheless, the very low FRET value obtained for mixtures with a POPC content of $40 \%$ suggests the presence of domains larger than the FRET infinite phase separation limit of $50 \mathrm{~nm}^{40}$ It should be stressed that in case phase separation resulted in domains larger than this limit or if domain fission occurred, no additional changes in FRET efficiencies would be expected.

The same methodology was applied to the mixtures of DOW/POPC already studied by our group at the micron scale. ${ }^{15}$ Once again, a decrease in FRET efficiency was also recorded when POPC was included in the mixture, suggesting nanoscale phase separation. Figure 8 shows all FRET efficiency values for the mixtures of copolymers and POPC as a function of POPC.

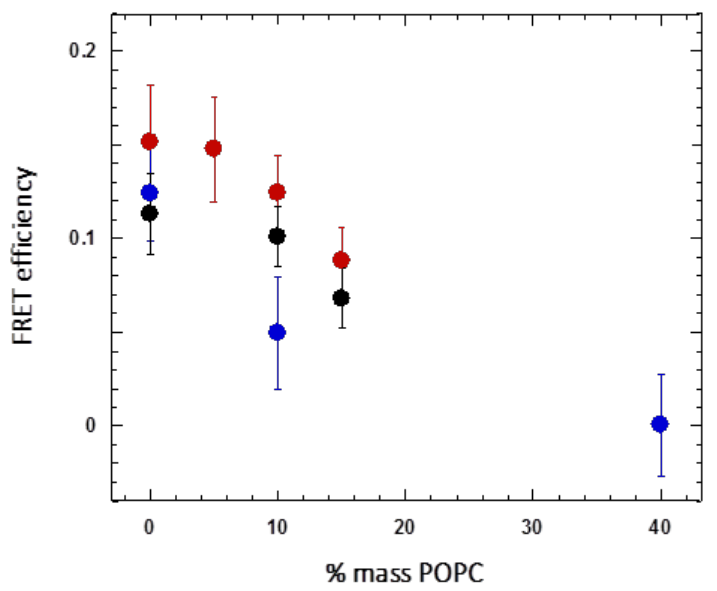

Figure 8. FRET efficiency in GHVs of POPC with $1.5 \mathrm{~K}(\bullet)$; DOW $(\bullet)$ and $3 \mathrm{~K}(\bullet)$ measured by FRET-FLIM methodology 
This document is the Accepted Manuscript version of an article that appeared in final form in Soft Matter, 2017, 13(3), 627-637, after peer review and technical editing by the publisher. To access the final edited and published work see DOI: 10.1039/C6SM01625A

Formation of nanodomains was suggested in GHUVs of PBut- $b$ PEO/DPPC, ${ }^{37}$ would result from nucleation growth mechanism modulated by cooling rate speed, but no direct proof could be obtained. Here, the origin of the POPC nanodomains is different as POPC is always in the fluid state, and our results demonstrate for the first time the formation of nanometric heterogeneities within hybrid polymer/lipid giant vesicles at low lipid content, where optical microscopy was previously unable to detect phase separation. Global results are finally summarized as "apparent" phase diagrams for each pair of copolymer and phospholipid (See Figures 9 and 10). Notice that due to fission of pure liposomes shortly after electroformation, these apparent phase diagrams do not fully correspond to the real thermodynamic phase diagrams for these mixtures but only reflect the phase composition of hybrid vesicles.

\section{Micrometer scale fluid/gel phase separation in GHUVs}

In order to explore the formation of gel-like domains in GHUVs, phase separation on GHUVs was also characterized on mixtures of the copolymers with DPPC as lipid. As the phase transition of DPPC is about $41^{\circ} \mathrm{C}$, measurements were carried either below or above this temperature. At $20^{\circ} \mathrm{C}$, just $5 \%$ of DPPC is enough to drive phase separation in mixtures with any of the triblock copolymers (images are shown in supporting information Figure S12)). It should be noted that DOPE-Rhod is excluded from the ordered phases, and PDMS-PEO copolymers present an extremely low miscibility in lipid phases as deduced from the partition coefficient extracted from fluorescence anisotropy measurements. ${ }^{16}$

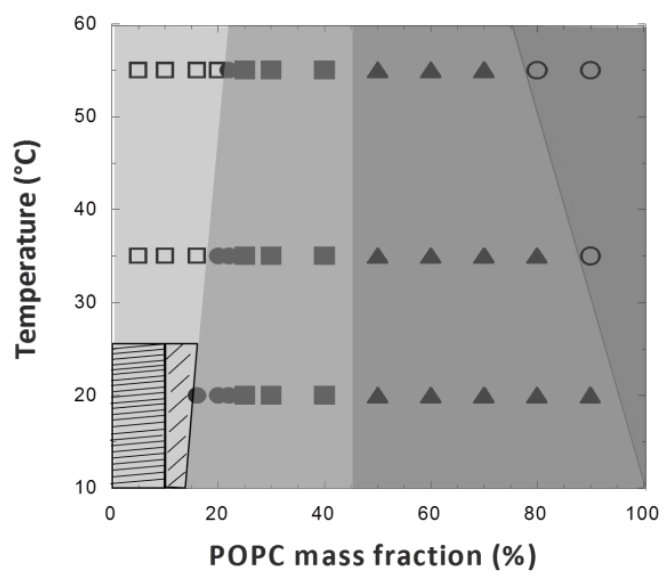

Figure 9. Outlined apparent phase diagram for 1.5K/POPC binary system determined by confocal microscopy and FLIM measurements. Symbols represent characterized fractions and temperatures by confocal microscopy; shaded areas depict regions of the phase diagram with and without macroscopic phase separation, as well as the stability of the coexisting domains. The hatched area represents the region where nanodomains were detected at $20^{\circ} \mathrm{C}$. $\square$ : No macroscopic domains. Fraction of vesicles exhibiting phase separation lower than $50 \%$ Fraction of vesicles exhibiting phase separation higher than $50 \%$. $\Delta$ Stable domain budding. O: Fission of budded domains.

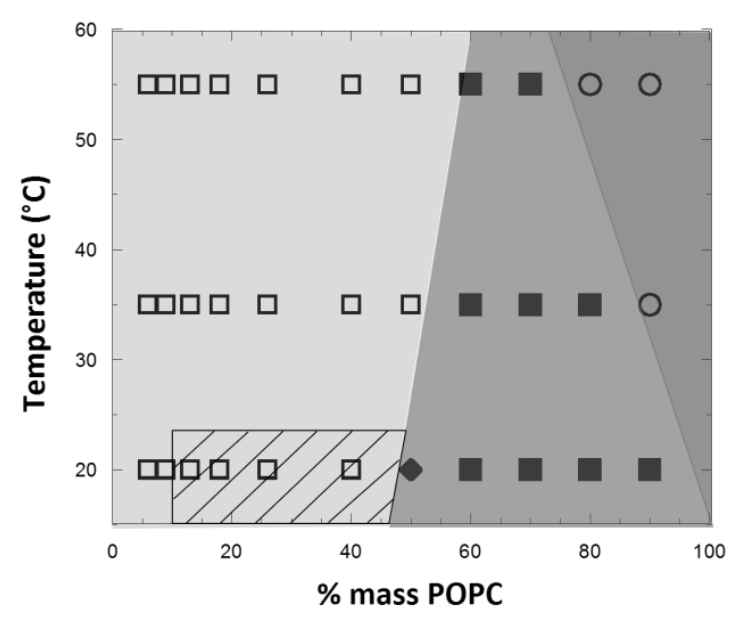

Figure 10. Outlined apparent phase diagram for the 3K/POPC binary system determined by confocal microscopy and FLIM measurements. Symbols represent characterized fractions and temperatures by confocal microscopy; shaded areas depict regions of the phase diagram with and without macroscopic phase separation, as well as the stability of the coexisting domains. The hatched area represents the region where nanodomains were detected at $20^{\circ} \mathrm{C}$. $\square$ : No macroscopic domains . Fraction of vesicles exhibiting phase separation lower than $50 \%$ Fraction of vesicles exhibiting phase separation higher than $50 \%$. $\Delta$ Stable domain budding. $\bigcirc$ : Fission of budded domains.

Thus the DPPC-gel domains are recognizable as dark domains, excluding both lipid and copolymer fluorescent analogues. While fluid-state lipid domains normally present spherical shapes, these dark DPPC gel-domains exhibited various morphologies depending on the cooling rate after electroformation at $50^{\circ} \mathrm{C}$.

It has been shown in literature that cooling rate has an effect on the membrane tension as it induces a thermal contraction of both membrane and water, the contraction of membrane being faster than the aqueous core of the vesicle, inducing membrane stress which can goes through a maximum. ${ }^{28}$ Indeed at high cooling rate, the rapid contraction of the membrane compared to the aqueous compartment abruptly reduces the surface-to-volume ratio and thus induces lateral tension in the membrane that can go above its lysis tension, inducing rupture and reseal phenomena during which water escapes from the vesicle, therefore relaxing tension. At low cooling rate, the membrane tension is continuously relaxed by natural diffusion of water through the membrane. ${ }^{32,41}$ The differences in membrane tension can then modulate the morphology of the DPPC domains (Patches versus stripes). We have therefore tried to evaluate the effect of the hydrophobic length mismatch that is modulated through the molar mass of the block copolymer at different cooling rates on the morphology and size of the domains obtained. Concretely, results are shown in figure 11 for hybrid vesicles obtained from the same electroformation process but cooled to $20^{\circ} \mathrm{C}$ at different rates, namely: $1^{\circ} \mathrm{C} / \mathrm{min}, 5^{\circ} \mathrm{C} / \mathrm{min}$ and $20^{\circ} \mathrm{C} / \mathrm{min}$. We also used a spontaneous cooling as it is the simplest procedure that can be used: It consists in allowing for cooling down naturally of the $1 \mathrm{~mL}$ vesicle suspension in a room 
This document is the Accepted Manuscript version of an article that appeared in final form in Soft Matter, 2017, 13(3), 627-637, after peer review and technical editing by the publisher. To access the final edited and published work see DOI: 10.1039/C6SM01625A

thermostated at $22^{\circ} \mathrm{C}$ just after its electroformation at $50^{\circ} \mathrm{C}$, in a closed Eppendorf tube. . The decrease of temperature for this procedure was measured (Figure S14) and can be considered to be reproducible and linear with a constant slope of $2.9^{\circ} \mathrm{C} / \mathrm{min}$ from $50^{\circ} \mathrm{C}$ until $35^{\circ} \mathrm{C}$.

Images shown on Fig.11 are representative of domain shapes obtained at each cooling rate. Natural cooling rate resulted in the formation of stripped gel domains while a controlled fast rate gave rise to many small patchy gel domains, and a controlled slow rate generated large star- or flower-like shaped domains. We had exactly the same phase behaviour described above for DOW, $1.5 \mathrm{~K}$ or $3 \mathrm{~K}$ copolymers. However, for $5 \mathrm{~K}$, only one type of morphology (patchy domain) was observed.

We have therefore tried to characterize the copolymer/DPPC GHUV behaviour when submitted to gradual heating up to $50^{\circ} \mathrm{C}$, well-above $\mathrm{Tm}$ of DPPC, to obtain a lipid fluid phase and compare the results obtained with copolymer/POPC mixtures.

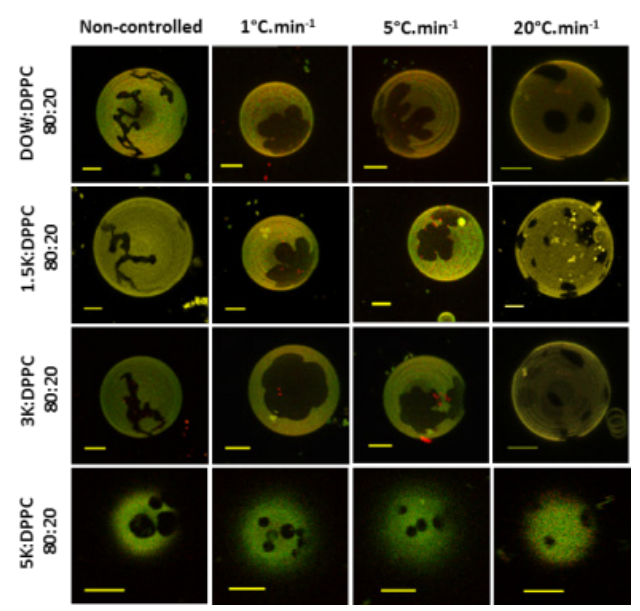

Figure 11. The impact of cooling rate on DPPC gel-domain morphology within phase separated DPPC/triblock copolymer GHUVs. Scale bar: $5 \mu \mathrm{m}$.

Briefly, for $1.5 \mathrm{~K} / \mathrm{DPPC}$ and DOW/DPPC mixtures, at $50^{\circ} \mathrm{C}$ no evidence of phase coexistence was detected for DPPC fractions below $15 \% \mathrm{w} / \mathrm{w}$ as it was observed for the copolymer/POPC mixtures at room temperature. For higher lipid content, separated DPPC-rich fluid phases and polymer-rich phases were observed, but no budding/fission phenomenon occurred for any of the DOW/DPPC mixtures. Interestingly, the structuration of those copolymer/DPPC GHUVs was found to be reversible upon temperature. As shown on Figure 12, large DPPC dark gel-state domains with irregular faceted shapes were observed at $20^{\circ} \mathrm{C}$, then they underwent a transition to circular red domains (enriched in DOPE-Rhod) when samples were heated up to $50^{\circ} \mathrm{C}$ : This transition reflects the minimization of boundary line energy allowed by the fluid character of the lipid phase, with progressive incorporation of DOPE-Rhod inside. Subsequent cooling and heating cycles reproduced these results.
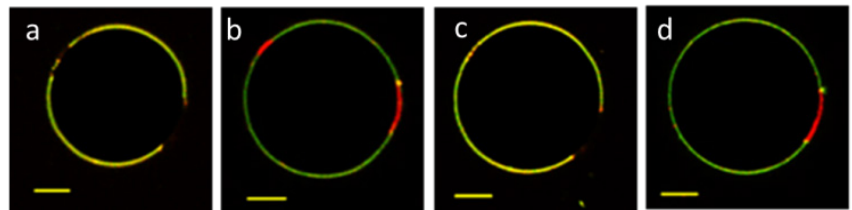

Figure 12. Reversible changes in phase separated GHVs of $1.5 \mathrm{~K} / \mathrm{DPPC} 80 / 20$ during cooling and heating cycles. A: $20^{\circ} \mathrm{C}$; b: heated up at $50^{\circ} \mathrm{C}$. c: back to $20^{\circ} \mathrm{C}$. d: re-heated up at $50^{\circ} \mathrm{C}$. Scale bar: $10 \mu \mathrm{m}$

For the triblock copolymer $3 \mathrm{~K}$, when DPPC was in a fluid state (above $41^{\circ} \mathrm{C}$ ), no phase separation was observed for samples with less than $50 \%$ DPPC content. Budding and fission of DPPCrich vesicles was observed at $50^{\circ} \mathrm{C}$ in samples containing higher fractions of DPPC (Figure 13) in analogy with what was observed for the 3K/POPC mixtures. Nevertheless, some DPPCrich domains were stable in the time-course of the experiment and after cooling they underwent transition to dark gel-state domains. No phase separation was observed in GHUVs of DPPC and triblock $5 \mathrm{~K}$ at $50^{\circ} \mathrm{C}$
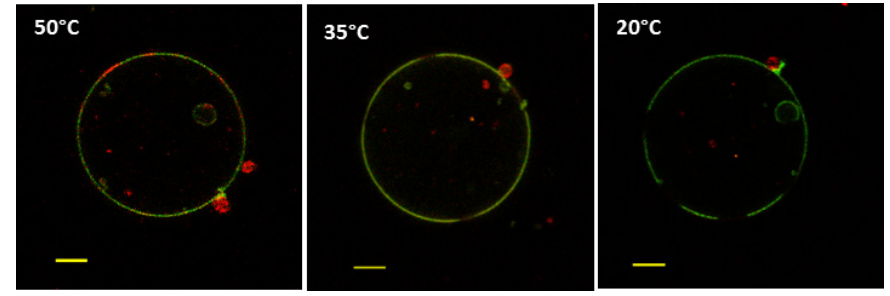

Figure 13 Hybrid vesicle obtained from 3K/DPPC 50/50 mixture observed at different temperatures: Some fluid-DPPC domains appeared at $50^{\circ} \mathrm{C}$ and formed gel-DPPC domains upon cooling, which progressively excluded the Rhod-DOPE dye at $35^{\circ} \mathrm{C}$ and $20^{\circ} \mathrm{C}$. The scale bar lengths are $5 \mu \mathrm{m}$.

\section{Discussions}

\section{Effect of hydrophobic mismatch}

Phase separation properties in GHUVs formed from mixtures of POPC and triblock copolymers were highly dependent on the copolymer block molar masses. This point was evidenced through comparison of their phase diagrams (Fig 10 and 11). Regarding the threshold of POPC content above which micron scale phase separation occurs, 16 weight $\%$ was necessary for $1.5 \mathrm{~K} / \mathrm{POPC}$ mixture, 50 weight $\%$ for $3 \mathrm{~K} / \mathrm{POPC}$, and no phase coexistence was detected for $5 \mathrm{~K} / \mathrm{POPC}$ mixtures. Nevertheless, submicron lipid domains were surprisingly detected from the FRET efficiency in FLIM experiments for low lipid concentrations not only in 1.5K/POPC GHVs but also in $3 \mathrm{~K} / \mathrm{POPC}$ GHVs. A higher hydrophobic mismatch is expected for the $3 \mathrm{~K}$ compared to the $1.5 \mathrm{~K}$ leading to a higher exposure of hydrophobic copolymer core to the aqueous environment. Since this exposure is associated with a large energetic cost, the line tension is expected to be higher in 3K/POPC hybrid membranes, and a larger tendency for domain coalescence and formation of larger domains was expected for this mixture, since this allows for the minimisation of the total boundary length. When the energetic costs associated with line tension are too high, the length of the interface can be 
This document is the Accepted Manuscript version of an article that appeared in final form in Soft Matter, 2017, 13(3), 627-637, after peer review and technical editing by the publisher. To access the final edited and published work see DOI: 10.1039/C6SM01625A

further minimized through membrane curvature (budding), as predicted by theory, ${ }^{14}$ which can ultimately evolve towards fission. This was indeed observed for 3K/POPC mixtures at lipid fractions below $50 \%$, for which rapid budding and fission phenomena lead to separated liposomes and hybrid homogeneous vesicles

On the other hand, a great number of vesicles at POPC content higher than 50 weight \% showed stable phase coexistence (after overnight incubation). This discrepancy between the stability of phase coexistence in 3K/POPC mixtures with low and high lipid content may be explained by differences in curvature energies of lipid-rich and polymer-rich domains. At high lipid content, the curvature of the polymer-rich membrane induced by the budding phenomenon is more pronounced and the bending energy associated with further budding is higher than the one associated with lipid domains. In addition, bending rigidity of polymer domains is larger than that of lipid domains as it is quadratic with the bilayer thickness. ${ }^{42}$ An equilibrium between line tension and bending energy costs ${ }^{23}$ is more easily achieved for small polymer-rich domains, while small lipid-rich domains easily undergo large curvatures increases followed by membrane fission into separated vesicles.

Using the same rationalization, we believe that the curved domains in 1.5K/POPC GHUVs are stable after days because the low hydrophobic length mismatch between $1.5 \mathrm{~K}$ and POPC leads to line energies lower than the membrane bending energy required for fission. And conversely for the case of $5 \mathrm{~K}$, given the extremely high hydrophobic length mismatch between polymer and lipid molecules, even if phasecoexistence is present during electroformation, these domains are extremely unstable and quickly disappear or evolve towards fission before their imaging is possible.

\section{Effect of copolymer architecture}

In this study, two copolymers (one commercial called "DOW" and the $1.5 \mathrm{~K}$ synthesized in our lab) of same chemical nature and hydrophobic membrane thickness ( $5 \mathrm{~nm})$ have similar molar masses and only differ by their architecture: grafted for DOW and triblock for $1.5 \mathrm{~K}$. Therefore the chain organization of the membrane is probably different. Regarding previous results of our group and of literature, the DOW is probably organized as a bilayer in analogy to phospholipids ${ }^{43}$ whereas hairpin or/and extended conformations can be found in the membrane for $1.5 \mathrm{~K}$, but this has not been quantified yet. ${ }^{44}$ Similar behaviours were observed for both copolymers, but in the case of DOW, lipid micrometric domains were only visible starting from $22 \%(\mathrm{w} / \mathrm{w})$ in lipid $(50 / 50 \mathrm{~mol})$ and above, whereas ${ }^{15}$ only 16 weight $\%$ in lipid fraction was enough to observe the phase coexistence in the case of $1.5 \mathrm{~K}$. Moreover, DOW/POPC mixtures displayed a clear instability of the domains (budding and fission) on the first few hours after electroformation, while the triblock $1.5 \mathrm{~K}$ showed a stable state for a few days. As the GHUVs were obtained in the same experimental conditions, such differences could be explained by different values of the line tension at the polymer/lipid boundaries and/or of the bending rigidity of the copolymer membrane. These parameters as well as the identification of chain conformation in these membranes need to be quantified in further studies. It is important to recall that even though budding and fission occurred, leading to the formation of separate liposomes and hybrid homogeneous vesicles, the presence of lipid nano-domains in these GHUVs was proven by FLIM/FRET experiments. The nano-domains are most probably in a metastable state that may lead later to growth at a micrometre size observable by optical microscopy.

As a last qualitative evidence of the metastable state of these nanodomains and the role of membrane tension on the structuration, we were able to observe the appearance of micrometric lipid rich domains under applied lateral tension, using micropipette suction, as illustrated in the Figure 14. This technique has already been used to show that membrane tension decrease temperature at which coexisting liquidordered (Lo) and liquid disordered (Ld) phase appear in lipid vesicle. ${ }^{45}$ We believe the apparition of microdomains is due to an increase of the line tension for lipid-polymer boundaries under membrane tension, as proposed by Akimov et al. ${ }^{46}$ Microdomains are therefore formed to limit the boundary line energy. When suction pressure is released, the microdomains remain, attesting the metastable state of the nanodomains.
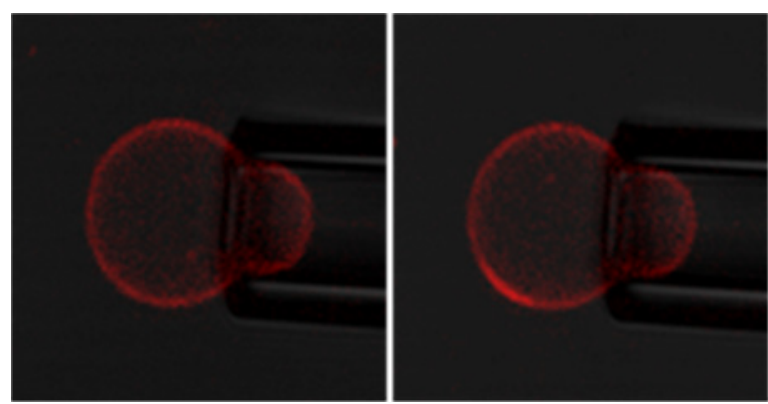

Figure 14: Appearance of a lipid microdomain (rich in RhodDOPE) under increased suction pressure for 3K/POPC (70/30 $\mathrm{w} / \mathrm{w} \%)$. No fluorescent labelled graft copolymer was included in these experiments. (left: applied tension $0.11 \mathrm{mN} / \mathrm{m}$, right: $0.22 \mathrm{mN} / \mathrm{m}$ )

\section{Effect of lipid fluidity}

In the studied POPC/copolymer mixtures, numerous differences have been observed between the different copolymers, explained by the modulation of hydrophobic length mismatch, line tension and bending rigidity of the membrane. With DPPC as lipid, the behaviours obtained looked similar: $5 \%$ of DPPC was enough to obtain stable lipid micron-sized domains in all studied mixtures. No budding phenomena were observed due to the solid character of the gel lipid phase. Interestingly, a lot of similarities with copolymer/POPC mixtures were observed for temperatures above the Tm of DPPC, with the exception of fission which was not observed, suggesting a slightly lower line tension at the lipid polymer boundaries compared to POPC. 
This document is the Accepted Manuscript version of an article that appeared in final form in Soft Matter, 2017, 13(3), 627-637, after peer review and technical editing by the publisher. To access the final edited and published work see DOI: 10.1039/C6SM01625A

Regarding the morphologies obtained at different cooling rates, some similarities were obtained with previous results of literature on PBut- $b$-PEO/DPPC mixtures observed at different cooling rates comparable to this study. Fast cooling favours the apparition of multiple small patchy domains ${ }^{37}$ as it reduces the free energy barrier for nucleation. Another aspect to consider is the membrane tension that can be modulated via the cooling rate as explained previously by difference of thermal dilatation coefficients between water and membranes. ${ }^{32}$ High membrane tension would favour stripe-like morphology of DPPC domains whereas low lateral tension would favour quasispherical patches. ${ }^{32}$ Stripes were observed systematically for "natural" cooling evaluated at $-2.9^{\circ} \mathrm{C} / \mathrm{min}$ from $50^{\circ} \mathrm{C}$ to $35^{\circ} \mathrm{C}$, for all copolymers excepted for $5 \mathrm{~K}$. Slightly slower or faster cooling gave rise to large flower-like domains, and small patchy domains were obtained at high cooling rate. In analogy with the work of Santore and col. ${ }^{32}$, it suggests that membrane tension goes to higher values for the "natural" cooling process. During slower cooling, the membrane stress can relax as water can diffuse across the membrane, whereas higher cooling rate generates tension that can overcome membrane lysis tension leading to rupture and reseal process (transient pores) during which water escapes from the vesicle and relaxes tension. However the modulation of domain morphologies has not been observed for the thickest $5 \mathrm{~K}$ copolymer. It is important to recall that line tension at the polymer /lipid boundary is higher for this system where the hydrophobic length mismatch is maximal. Moreover, membrane tension can lead to an increase of the line tension, as it will alter the deformation of the monolayer that occurs at the lipid poymer/interface to avoid hydrophobic exposure to water. ${ }^{46}$ Therefore the formation of stripe morphology is unlikely as this would results in higher boundary line energies compared to patchy domains.

\section{Conclusions}

In this work; we reported a systematic study on GHUV formation using copolymers with same chemical nature based on PDMS as hydrophobic block and PEO as hydrophilic block, but with different architectures (grafted versus triblock) and molar masses. They were blended with phosphocholine lipids at either gel or fluid lipid phase at room temperature. By using a combination of classical confocal microscopy imaging with an advanced fluorescence microscopy technique (FLIM-FRET), we were able to probe membrane structure at both the microand nanoscale. In particular, we brought a direct spectroscopic evidence of the presence of nano-domains in GHUVs, which until now had only been suggested. ${ }^{37}$ Globally, the study clearly shows the effect of the modulation of the line tension as well as bending rigidity of the polymer membrane on the GHUV morphology that could be obtained with a phospholipid in the fluid lipid phase at ambient temperature. The stabilization of fluid-state lipid micron-sized domains can occur in the membrane above a given threshold lipid fraction for copolymer presenting low molar mass and with a membrane thickness close to the bilayer of liposomes. Very interestingly, stable budded vesicles illustrating equilibrium between domain boundary energy and membrane curvature energy can be obtained, depending on the molar mass of the copolymer and on the lipid fraction. In addition, we showed that homogenous hybrid vesicles at the micron scale can only be observed below a given lipid fraction. This results from the budding and fission of lipid domains which occurs rapidly after electroformation for polymers and lipids with a high hydrophobic mismatch in the membrane. A fraction of the lipid remains in the polymer membrane, apparently as nanodomains which are probably highly stable. Below 10 weight \% (for $1.5 \mathrm{~K}$ and $3 \mathrm{~K} / \mathrm{POPC}$ mixtures) the lipid was homogenously dispersed in the polymer membrane. Interestingly, the molar mass effect of the copolymer on membrane structuration can also be detected with lipid in the gel state at room temperature (DPPC), although less variation was observed compared to formulations with lipid in the fluid phase (POPC). If different morphologies can be obtained by modulation of the cooling rate and therefore induced membrane tension as interpreted by Chen and Santore, ${ }^{32}$ it seems that a very high hydrophobic length mismatch drives the morphology towards patchy domains whatever the cooling process used. It is worth mentioning that the library of systems presented here will be used to quantify crucial parameters such as line tension at the lipid/polymer boundary and the bending rigidity through the help of micropipette aspiration techniques. The outlook of this study will be to extend the qualitative interpretation in literature by more quantitative measurements of these two parameters: line tension and bending rigidity of the respectively lipid-rich and polymer-rich domains. This will be particularly helpful to understand more precisely the origin of membrane structuration at different cooling speed and confirm the mechanism proposed or open discussion on other mechanisms suggested on multicomponent lipid vesicle like Ostwald's rule of stage. ${ }^{47}$

\section{Acknowledgements}

Funding: International doctoral school on Functional Materials (IDS-FunMat), Erasmus Mundus (EU), FCT (FAPESP/20107/2014, UID/NAN/50024/2013 and RECI/CTMPOL/0342/2012), and Agence Nationale de la Recherche (ANR 12-BS08-0018 KBT).

M. Er Rafik and M. Schmutz from Institut Charles Sadron (UPR CNRS 22, Strasbourg) for CryoTEM imaging of pure polymersomes

\section{Notes and references}

1. J. F. Le Meins, C. Schatz, S. Lecommandoux and O. Sandre, Materials Today, 2013, 16, 397-402.

2. M. Schulz and W. H. Binder, Macromol Rapid Commun, 2015, 36, 2031-2041.

3. W. H. Binder, V. Barragan and F. M. Menger, Angewandte Chemie, International Edition, 2003, 42, 5802-5827. 
This document is the Accepted Manuscript version of an article that appeared in final form in Soft Matter, 2017, 13(3), 627-637, after peer review and technical editing by the publisher. To access the final edited and published work see DOI: 10.1039/C6SM01625A

4. R. Lipowsky and R. Dimova, Journal of Physics-Condensed Matter, 2003, 15, S31-S45.

5. A. J. Garcia-Saez and P. Schwille, FEBS Letters, 2010, 584 1653-1658.

6. V. A. Frolov, Y. A. Chizmadzhev, F. S. Cohen and J. Zimmerberg, Biophys J, 2006, 91, 189-205.

7. T. S. Ursell, W. S. Klug and R. Phillips, Proc Natl Acad Sci U S A, 2009, 106, 13301-13306.

8. A. Travesset, J Chem Phys, 2006, 125, 084905.

9. S. Semrau, T. Idema, T. Schmidt and C. Storm, Biophys $J$, 2009, 96, 4906-4915.

10. J. Hu, T. Weikl and R. Lipowsky, Soft Matter, 2011, 7, 60926102.

11. F. Brochard-Wyart and P. G. de Gennes, Proceedings of the National Academy of Sciences, 2002, 99, 7854-7859.

12. L. Bagatolli and P. B. S. Kumar, Soft Matter, 2009, 5, 32343248.

13. P. I. Kuzmin, S. A. Akimov, Y. A. Chizmadzhev, J. Zimmerberg and F. S. Cohen, Biophys J, 2005, 88, 1120-1133.

14. J. Wolff, S. Komura and D. Andelman, Phys Rev E Stat Nonlin Soft Matter Phys, 2015, 91, 012708.

15. M. Chemin, P. M. Brun, S. Lecommandoux, O. Sandre and J. F. Le Meins, Soft Matter, 2012, 8, 2867-2874.

16. T. P. T. Dao, F. Fernandes, M. Er-Rafik, R. Salva, M. Schmutz, A. Brulet, M. Prieto, O. Sandre and J. F. Le Meins, ACS Macro Letters, 2015, 4, 182-186.

17. T. Ruysschaert, A. F. P. Sonnen, T. Haefele, W. Meier, M. Winterhalter and D. Fournier, Journal of the American Chemical Society, 2005, 127, 6242-6247.

18. Z. Cheng, D. R. Elias, N. P. Kamat, E. Johnston, A. A. Poloukhtine, V. V. Popik, D. A. Hammer and A. Tsourkas, Bioconjugate Chemistry, 2011, 22, 2021-2029.

19. J. Nam, P. A. Beales and T. K. Vanderlick, Langmuir, 2011, 27, 1-6.

20. S. Lim, H.-P. de Hoog, A. Parikh, M. Nallani and B. Liedberg, Polymers, 2013, 5, 1102-1114.

21. A. Olubummo, M. Schulz, R. SchÃ pps, J. r. Kressler and W. H. Binder, Langmuir, 2014, 30, 259-267.

22. R. Lipowsky, J. Phys. II France, 1992, 2, 1825-1840.

23. R. Lipowsky, Biophysical Journal 1993, 64, 1133-1138.

24. F. Jülicher and R. Lipowsky, Physical Review Letters, 1993, 70, 2964-2967.

25. F. Jülicher and R. Lipowsky, Physical Review E, 1996, 53, 2670-2683.

26. M. Schulz, A. Olubummo, K. Bacia and W. H. Binder, Soft Matter, 2014, 10, 831-839.

27. J. F. Nagle and S. Tristram-Nagle, Biochimica et Biophysica Acta (BBA) - Reviews on Biomembranes, 2000, 1469, 159195.

28. A. Carlsen, N. Glaser, J.-F. Le Meins and S. Lecommandoux, Langmuir, 2011, 27, 4884-4890.

29. Z. Lin, R. M. Hill, H. T. Davis, L. E. Scriven and Y. Talmon, Langmuir, 1994, 10, 1008-1011.

30. R. M. Hill, Langmuir, 1993, 9, 2789-2798.

31. M. I. Angelova and D. S. Dimitrov, Faraday Discussions of the Chemical Society, 1986, 81, 303-311.
32. D. Chen and M. M. Santore, Soft Matter, 2015, 11, 26172626.

33. M. Schulz, S. Werner, K. Bacia and W. H. Binder, Angew Chem Int Ed Engl, 2013, 52, 1829-1833.

34. M. Schulz, D. Glatte, A. Meister, P. Scholtysek, A. Kerth, A. Blume, K. Bacia and W. H. Binder, Soft Matter, 2011, 7, 81008110.

35. A. Olubummo, M. Schulz, B.-D. Lechner, P. Scholtysek, K. Bacia, A. Blume, J. Kressler and W. H. Binder, ACS nano, 2012, 6, 8713-8727.

36. Z. Cheng and A. Tsourkas, Langmuir, 2008, 24, 8169-8173.

37. J. Nam, T. K. Vanderlick and P. A. Beales, Soft Matter, 2012, 8, 7982-7988.

38. R. Sachl, J. Humpolickova, M. Stefl, L. B. Johansson and M. Hof, Biophys J, 2011, 101, L60-62.

39. K. B. Towles and N. Dan, Langmuir, 2007, 23, 4737-4739.

40. L. M. S. Loura, F. Fernandes and M. Prieto, European Biophysics Journal with Biophysics Letters, 2010, 39, 589-607.

41. D. Chen and M. M. Santore, Proceedings of the National Academy of Sciences of the United States of America, 2014, 111, 179-184.

42. E. A. Evans, 1974, 14, 923-931.

43. R. Salva, J.-F. Le Meins, O. Sandre, A. Brûlet, M. Schmutz, P. Guenoun and S. Lecommandoux, ACS Nano, 2013, 7, 92989311.

44. F. Itel, M. Chami, A. Najer, S. Loercher, D. Wu, I. A. Dinu and W. Meier, Macromolecules, 2014, 47, 7588-7596.

45. T. Portet, S. E. Gordon and S. L. Keller, Biophysical Journal, 2012, 103, L35-L37.

46. S. A. Akimov, P. I. Kuzmin, J. Zimmerberg and F. S. Cohen, Phys Rev E Stat Nonlin Soft Matter Phys, 2007, 75, 011919.

47. V. D. Gordon, P. A. Beales, Z. Zhao, C. Blake, F. C. MacKintosh, P. D. Olmsted, M. E. Cates, S. U. Egelhaaf and W. C. K. Poon, Journal of Physics-Condensed Matter, 2006, 18, L415-L420. 